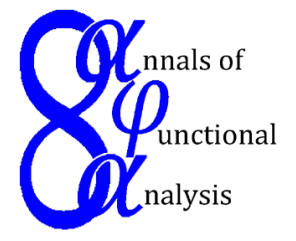

Ann. Funct. Anal. 7 (2016), no. 1, 42-60

http://dx.doi.org/10.1215/20088752-3163513

ISSN: 2008-8752 (electronic)

http://projecteuclid.org/afa

\title{
THE CONE AND CYLINDER ALGEBRAS
}

\author{
RAYMOND MORTINI $^{1^{*}}$ and RUDOLF RUPP ${ }^{2}$ \\ Dedicated to Professor Anthony To-Ming Lau
}

Communicated by J. Esterle

\begin{abstract}
In this exposition-type note we present detailed proofs of certain assertions concerning several algebraic properties of the cone and cylinder algebras. These include a determination of the maximal ideals, the solution of the Bézout equation, and a computation of the stable ranks by elementary methods.
\end{abstract}

\section{INTRODUCTION}

Let $\mathbb{D}=\{z \in \mathbb{C}:|z|<1\}$ be the open unit disk in the complex plane $\mathbb{C}$, and let $\mathbf{D}$ be its closure. As usual, $C(\mathbf{D}, \mathbb{C})$ denotes the space of continuous, complexvalued functions on $\mathbf{D}$ and $A(\mathbf{D})$ denotes the disk algebra, that is, the algebra of all functions in $C(\mathbf{D}, \mathbb{C})$ which are holomorphic in $\mathbb{D}$. By the Stone-Weierstrass theorem, we have $C(\mathbf{D}, \mathbb{C})=[z, \bar{z}]_{\text {alg }}$ and $A(\mathbf{D})=[z]_{\text {alg }}$, the uniformly closed subalgebras generated by $z, \bar{z}$ (resp., $z$ ) on $\mathbf{D}$. In this expositional note, we study the uniformly closed subalgebra

$$
A_{c o}=[z,|z|]_{\mathrm{alg}} \subseteq C(\mathbf{D}, \mathbb{C})
$$

of $C(\mathbf{D}, \mathbb{C})$, which is generated by $z$ and $|z|$, as well as by the algebra

$$
\operatorname{Cyl}(\mathbb{D})=\{f \in C(\mathbf{D} \times[0,1], \mathbb{C}): f(\cdot, t) \in A(\mathbf{D}) \text { for all } t \in[0,1]\} .
$$

We will call the algebra $A_{c o}$ the cone algebra and the algebra $\mathrm{Cyl}(\mathbb{D})$ the cylinder algebra.

Copyright 2016 by the Tusi Mathematical Research Group.

Received Dec. 4, 2014; Accepted May 13, 2015.

${ }^{*}$ Corresponding author.

2010 Mathematics Subject Classification. Primary 46J10; Secondary 46J15, 46J20, 30H50.

Keywords. cone algebra, cylinder algebra, Bézout equation, maximal ideals, stable ranks. 


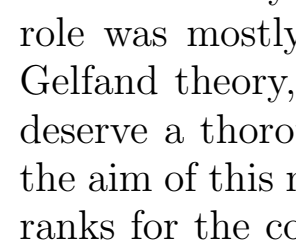

Ann. Funct. Anal. 7 (2016), no. 1, 42-60

http://dx.doi.org/10.1215/20088752-3163513

ISSN: 2008-8752 (electronic)

http://projecteuclid.org/afa

\title{
THE CONE AND CYLINDER ALGEBRAS
}

\author{
RAYMOND MORTINI $^{1^{*}}$ and RUDOLF RUPP ${ }^{2}$ \\ Dedicated to Professor Anthony To-Ming Lau
}

Communicated by J. Esterle

\begin{abstract}
In this exposition-type note we present detailed proofs of certain assertions concerning several algebraic properties of the cone and cylinder algebras. These include a determination of the maximal ideals, the solution of the Bézout equation, and a computation of the stable ranks by elementary methods.
\end{abstract}

\section{INTRODUCTION}

Let $\mathbb{D}=\{z \in \mathbb{C}:|z|<1\}$ be the open unit disk in the complex plane $\mathbb{C}$, and let $\mathbf{D}$ be its closure. As usual, $C(\mathbf{D}, \mathbb{C})$ denotes the space of continuous, complexvalued functions on $\mathbf{D}$ and $A(\mathbf{D})$ denotes the disk algebra, that is, the algebra of all functions in $C(\mathbf{D}, \mathbb{C})$ which are holomorphic in $\mathbb{D}$. By the Stone-Weierstrass theorem, we have $C(\mathbf{D}, \mathbb{C})=[z, \bar{z}]_{\text {alg }}$ and $A(\mathbf{D})=[z]_{\text {alg }}$, the uniformly closed subalgebras generated by $z, \bar{z}$ (resp., $z$ ) on $\mathbf{D}$. In this expositional note, we study the uniformly closed subalgebra

$$
A_{c o}=[z,|z|]_{\mathrm{alg}} \subseteq C(\mathbf{D}, \mathbb{C})
$$

of $C(\mathbf{D}, \mathbb{C})$, which is generated by $z$ and $|z|$, as well as by the algebra

$$
\operatorname{Cyl}(\mathbb{D})=\{f \in C(\mathbf{D} \times[0,1], \mathbb{C}): f(\cdot, t) \in A(\mathbf{D}) \text { for all } t \in[0,1]\} .
$$

We will call the algebra $A_{c o}$ the cone algebra and the algebra $\mathrm{Cyl}(\mathbb{D})$ the cylinder algebra.

Copyright 2016 by the Tusi Mathematical Research Group.

Received Dec. 4, 2014; Accepted May 13, 2015.

${ }^{*}$ Corresponding author.

2010 Mathematics Subject Classification. Primary 46J10; Secondary 46J15, 46J20, 30H50.

Keywords. cone algebra, cylinder algebra, Bézout equation, maximal ideals, stable ranks. 


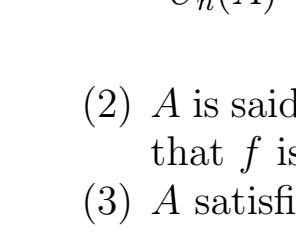

Ann. Funct. Anal. 7 (2016), no. 1, 42-60

http://dx.doi.org/10.1215/20088752-3163513

ISSN: 2008-8752 (electronic)

http://projecteuclid.org/afa

\title{
THE CONE AND CYLINDER ALGEBRAS
}

\author{
RAYMOND MORTINI $^{1^{*}}$ and RUDOLF RUPP ${ }^{2}$ \\ Dedicated to Professor Anthony To-Ming Lau
}

Communicated by J. Esterle

\begin{abstract}
In this exposition-type note we present detailed proofs of certain assertions concerning several algebraic properties of the cone and cylinder algebras. These include a determination of the maximal ideals, the solution of the Bézout equation, and a computation of the stable ranks by elementary methods.
\end{abstract}

\section{INTRODUCTION}

Let $\mathbb{D}=\{z \in \mathbb{C}:|z|<1\}$ be the open unit disk in the complex plane $\mathbb{C}$, and let $\mathbf{D}$ be its closure. As usual, $C(\mathbf{D}, \mathbb{C})$ denotes the space of continuous, complexvalued functions on $\mathbf{D}$ and $A(\mathbf{D})$ denotes the disk algebra, that is, the algebra of all functions in $C(\mathbf{D}, \mathbb{C})$ which are holomorphic in $\mathbb{D}$. By the Stone-Weierstrass theorem, we have $C(\mathbf{D}, \mathbb{C})=[z, \bar{z}]_{\text {alg }}$ and $A(\mathbf{D})=[z]_{\text {alg }}$, the uniformly closed subalgebras generated by $z, \bar{z}$ (resp., $z$ ) on $\mathbf{D}$. In this expositional note, we study the uniformly closed subalgebra

$$
A_{c o}=[z,|z|]_{\mathrm{alg}} \subseteq C(\mathbf{D}, \mathbb{C})
$$

of $C(\mathbf{D}, \mathbb{C})$, which is generated by $z$ and $|z|$, as well as by the algebra

$$
\operatorname{Cyl}(\mathbb{D})=\{f \in C(\mathbf{D} \times[0,1], \mathbb{C}): f(\cdot, t) \in A(\mathbf{D}) \text { for all } t \in[0,1]\} .
$$

We will call the algebra $A_{c o}$ the cone algebra and the algebra $\mathrm{Cyl}(\mathbb{D})$ the cylinder algebra.

Copyright 2016 by the Tusi Mathematical Research Group.

Received Dec. 4, 2014; Accepted May 13, 2015.

${ }^{*}$ Corresponding author.

2010 Mathematics Subject Classification. Primary 46J10; Secondary 46J15, 46J20, 30H50.

Keywords. cone algebra, cylinder algebra, Bézout equation, maximal ideals, stable ranks. 


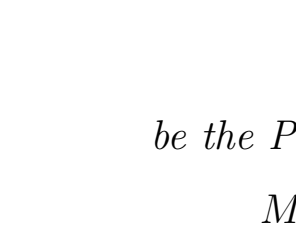

Ann. Funct. Anal. 7 (2016), no. 1, 42-60

http://dx.doi.org/10.1215/20088752-3163513

ISSN: 2008-8752 (electronic)

http://projecteuclid.org/afa

\title{
THE CONE AND CYLINDER ALGEBRAS
}

\author{
RAYMOND MORTINI $^{1^{*}}$ and RUDOLF RUPP ${ }^{2}$ \\ Dedicated to Professor Anthony To-Ming Lau
}

Communicated by J. Esterle

\begin{abstract}
In this exposition-type note we present detailed proofs of certain assertions concerning several algebraic properties of the cone and cylinder algebras. These include a determination of the maximal ideals, the solution of the Bézout equation, and a computation of the stable ranks by elementary methods.
\end{abstract}

\section{INTRODUCTION}

Let $\mathbb{D}=\{z \in \mathbb{C}:|z|<1\}$ be the open unit disk in the complex plane $\mathbb{C}$, and let $\mathbf{D}$ be its closure. As usual, $C(\mathbf{D}, \mathbb{C})$ denotes the space of continuous, complexvalued functions on $\mathbf{D}$ and $A(\mathbf{D})$ denotes the disk algebra, that is, the algebra of all functions in $C(\mathbf{D}, \mathbb{C})$ which are holomorphic in $\mathbb{D}$. By the Stone-Weierstrass theorem, we have $C(\mathbf{D}, \mathbb{C})=[z, \bar{z}]_{\text {alg }}$ and $A(\mathbf{D})=[z]_{\text {alg }}$, the uniformly closed subalgebras generated by $z, \bar{z}$ (resp., $z$ ) on $\mathbf{D}$. In this expositional note, we study the uniformly closed subalgebra

$$
A_{c o}=[z,|z|]_{\mathrm{alg}} \subseteq C(\mathbf{D}, \mathbb{C})
$$

of $C(\mathbf{D}, \mathbb{C})$, which is generated by $z$ and $|z|$, as well as by the algebra

$$
\operatorname{Cyl}(\mathbb{D})=\{f \in C(\mathbf{D} \times[0,1], \mathbb{C}): f(\cdot, t) \in A(\mathbf{D}) \text { for all } t \in[0,1]\} .
$$

We will call the algebra $A_{c o}$ the cone algebra and the algebra $\mathrm{Cyl}(\mathbb{D})$ the cylinder algebra.

Copyright 2016 by the Tusi Mathematical Research Group.

Received Dec. 4, 2014; Accepted May 13, 2015.

${ }^{*}$ Corresponding author.

2010 Mathematics Subject Classification. Primary 46J10; Secondary 46J15, 46J20, 30H50.

Keywords. cone algebra, cylinder algebra, Bézout equation, maximal ideals, stable ranks. 


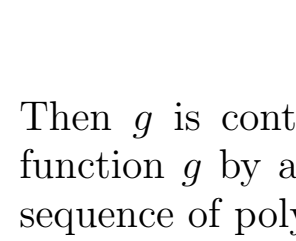

Ann. Funct. Anal. 7 (2016), no. 1, 42-60

http://dx.doi.org/10.1215/20088752-3163513

ISSN: 2008-8752 (electronic)

http://projecteuclid.org/afa

\title{
THE CONE AND CYLINDER ALGEBRAS
}

\author{
RAYMOND MORTINI $^{1^{*}}$ and RUDOLF RUPP ${ }^{2}$ \\ Dedicated to Professor Anthony To-Ming Lau
}

Communicated by J. Esterle

\begin{abstract}
In this exposition-type note we present detailed proofs of certain assertions concerning several algebraic properties of the cone and cylinder algebras. These include a determination of the maximal ideals, the solution of the Bézout equation, and a computation of the stable ranks by elementary methods.
\end{abstract}

\section{INTRODUCTION}

Let $\mathbb{D}=\{z \in \mathbb{C}:|z|<1\}$ be the open unit disk in the complex plane $\mathbb{C}$, and let $\mathbf{D}$ be its closure. As usual, $C(\mathbf{D}, \mathbb{C})$ denotes the space of continuous, complexvalued functions on $\mathbf{D}$ and $A(\mathbf{D})$ denotes the disk algebra, that is, the algebra of all functions in $C(\mathbf{D}, \mathbb{C})$ which are holomorphic in $\mathbb{D}$. By the Stone-Weierstrass theorem, we have $C(\mathbf{D}, \mathbb{C})=[z, \bar{z}]_{\text {alg }}$ and $A(\mathbf{D})=[z]_{\text {alg }}$, the uniformly closed subalgebras generated by $z, \bar{z}$ (resp., $z$ ) on $\mathbf{D}$. In this expositional note, we study the uniformly closed subalgebra

$$
A_{c o}=[z,|z|]_{\mathrm{alg}} \subseteq C(\mathbf{D}, \mathbb{C})
$$

of $C(\mathbf{D}, \mathbb{C})$, which is generated by $z$ and $|z|$, as well as by the algebra

$$
\operatorname{Cyl}(\mathbb{D})=\{f \in C(\mathbf{D} \times[0,1], \mathbb{C}): f(\cdot, t) \in A(\mathbf{D}) \text { for all } t \in[0,1]\} .
$$

We will call the algebra $A_{c o}$ the cone algebra and the algebra $\mathrm{Cyl}(\mathbb{D})$ the cylinder algebra.

Copyright 2016 by the Tusi Mathematical Research Group.

Received Dec. 4, 2014; Accepted May 13, 2015.

${ }^{*}$ Corresponding author.

2010 Mathematics Subject Classification. Primary 46J10; Secondary 46J15, 46J20, 30H50.

Keywords. cone algebra, cylinder algebra, Bézout equation, maximal ideals, stable ranks. 


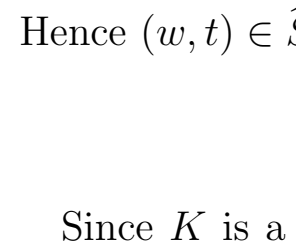

Ann. Funct. Anal. 7 (2016), no. 1, 42-60

http://dx.doi.org/10.1215/20088752-3163513

ISSN: 2008-8752 (electronic)

http://projecteuclid.org/afa

\title{
THE CONE AND CYLINDER ALGEBRAS
}

\author{
RAYMOND MORTINI $^{1^{*}}$ and RUDOLF RUPP ${ }^{2}$ \\ Dedicated to Professor Anthony To-Ming Lau
}

Communicated by J. Esterle

\begin{abstract}
In this exposition-type note we present detailed proofs of certain assertions concerning several algebraic properties of the cone and cylinder algebras. These include a determination of the maximal ideals, the solution of the Bézout equation, and a computation of the stable ranks by elementary methods.
\end{abstract}

\section{INTRODUCTION}

Let $\mathbb{D}=\{z \in \mathbb{C}:|z|<1\}$ be the open unit disk in the complex plane $\mathbb{C}$, and let $\mathbf{D}$ be its closure. As usual, $C(\mathbf{D}, \mathbb{C})$ denotes the space of continuous, complexvalued functions on $\mathbf{D}$ and $A(\mathbf{D})$ denotes the disk algebra, that is, the algebra of all functions in $C(\mathbf{D}, \mathbb{C})$ which are holomorphic in $\mathbb{D}$. By the Stone-Weierstrass theorem, we have $C(\mathbf{D}, \mathbb{C})=[z, \bar{z}]_{\text {alg }}$ and $A(\mathbf{D})=[z]_{\text {alg }}$, the uniformly closed subalgebras generated by $z, \bar{z}$ (resp., $z$ ) on $\mathbf{D}$. In this expositional note, we study the uniformly closed subalgebra

$$
A_{c o}=[z,|z|]_{\mathrm{alg}} \subseteq C(\mathbf{D}, \mathbb{C})
$$

of $C(\mathbf{D}, \mathbb{C})$, which is generated by $z$ and $|z|$, as well as by the algebra

$$
\operatorname{Cyl}(\mathbb{D})=\{f \in C(\mathbf{D} \times[0,1], \mathbb{C}): f(\cdot, t) \in A(\mathbf{D}) \text { for all } t \in[0,1]\} .
$$

We will call the algebra $A_{c o}$ the cone algebra and the algebra $\mathrm{Cyl}(\mathbb{D})$ the cylinder algebra.

Copyright 2016 by the Tusi Mathematical Research Group.

Received Dec. 4, 2014; Accepted May 13, 2015.

${ }^{*}$ Corresponding author.

2010 Mathematics Subject Classification. Primary 46J10; Secondary 46J15, 46J20, 30H50.

Keywords. cone algebra, cylinder algebra, Bézout equation, maximal ideals, stable ranks. 


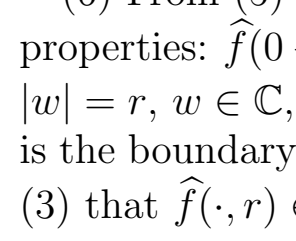

Ann. Funct. Anal. 7 (2016), no. 1, 42-60

http://dx.doi.org/10.1215/20088752-3163513

ISSN: 2008-8752 (electronic)

http://projecteuclid.org/afa

\title{
THE CONE AND CYLINDER ALGEBRAS
}

\author{
RAYMOND MORTINI $^{1^{*}}$ and RUDOLF RUPP ${ }^{2}$ \\ Dedicated to Professor Anthony To-Ming Lau
}

Communicated by J. Esterle

\begin{abstract}
In this exposition-type note we present detailed proofs of certain assertions concerning several algebraic properties of the cone and cylinder algebras. These include a determination of the maximal ideals, the solution of the Bézout equation, and a computation of the stable ranks by elementary methods.
\end{abstract}

\section{INTRODUCTION}

Let $\mathbb{D}=\{z \in \mathbb{C}:|z|<1\}$ be the open unit disk in the complex plane $\mathbb{C}$, and let $\mathbf{D}$ be its closure. As usual, $C(\mathbf{D}, \mathbb{C})$ denotes the space of continuous, complexvalued functions on $\mathbf{D}$ and $A(\mathbf{D})$ denotes the disk algebra, that is, the algebra of all functions in $C(\mathbf{D}, \mathbb{C})$ which are holomorphic in $\mathbb{D}$. By the Stone-Weierstrass theorem, we have $C(\mathbf{D}, \mathbb{C})=[z, \bar{z}]_{\text {alg }}$ and $A(\mathbf{D})=[z]_{\text {alg }}$, the uniformly closed subalgebras generated by $z, \bar{z}$ (resp., $z$ ) on $\mathbf{D}$. In this expositional note, we study the uniformly closed subalgebra

$$
A_{c o}=[z,|z|]_{\mathrm{alg}} \subseteq C(\mathbf{D}, \mathbb{C})
$$

of $C(\mathbf{D}, \mathbb{C})$, which is generated by $z$ and $|z|$, as well as by the algebra

$$
\operatorname{Cyl}(\mathbb{D})=\{f \in C(\mathbf{D} \times[0,1], \mathbb{C}): f(\cdot, t) \in A(\mathbf{D}) \text { for all } t \in[0,1]\} .
$$

We will call the algebra $A_{c o}$ the cone algebra and the algebra $\mathrm{Cyl}(\mathbb{D})$ the cylinder algebra.

Copyright 2016 by the Tusi Mathematical Research Group.

Received Dec. 4, 2014; Accepted May 13, 2015.

${ }^{*}$ Corresponding author.

2010 Mathematics Subject Classification. Primary 46J10; Secondary 46J15, 46J20, 30H50.

Keywords. cone algebra, cylinder algebra, Bézout equation, maximal ideals, stable ranks. 


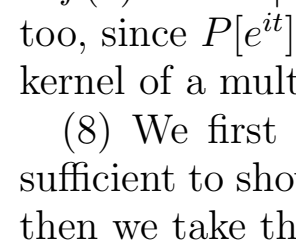

Ann. Funct. Anal. 7 (2016), no. 1, 42-60

http://dx.doi.org/10.1215/20088752-3163513

ISSN: 2008-8752 (electronic)

http://projecteuclid.org/afa

\title{
THE CONE AND CYLINDER ALGEBRAS
}

\author{
RAYMOND MORTINI $^{1^{*}}$ and RUDOLF RUPP ${ }^{2}$ \\ Dedicated to Professor Anthony To-Ming Lau
}

Communicated by J. Esterle

\begin{abstract}
In this exposition-type note we present detailed proofs of certain assertions concerning several algebraic properties of the cone and cylinder algebras. These include a determination of the maximal ideals, the solution of the Bézout equation, and a computation of the stable ranks by elementary methods.
\end{abstract}

\section{INTRODUCTION}

Let $\mathbb{D}=\{z \in \mathbb{C}:|z|<1\}$ be the open unit disk in the complex plane $\mathbb{C}$, and let $\mathbf{D}$ be its closure. As usual, $C(\mathbf{D}, \mathbb{C})$ denotes the space of continuous, complexvalued functions on $\mathbf{D}$ and $A(\mathbf{D})$ denotes the disk algebra, that is, the algebra of all functions in $C(\mathbf{D}, \mathbb{C})$ which are holomorphic in $\mathbb{D}$. By the Stone-Weierstrass theorem, we have $C(\mathbf{D}, \mathbb{C})=[z, \bar{z}]_{\text {alg }}$ and $A(\mathbf{D})=[z]_{\text {alg }}$, the uniformly closed subalgebras generated by $z, \bar{z}$ (resp., $z$ ) on $\mathbf{D}$. In this expositional note, we study the uniformly closed subalgebra

$$
A_{c o}=[z,|z|]_{\mathrm{alg}} \subseteq C(\mathbf{D}, \mathbb{C})
$$

of $C(\mathbf{D}, \mathbb{C})$, which is generated by $z$ and $|z|$, as well as by the algebra

$$
\operatorname{Cyl}(\mathbb{D})=\{f \in C(\mathbf{D} \times[0,1], \mathbb{C}): f(\cdot, t) \in A(\mathbf{D}) \text { for all } t \in[0,1]\} .
$$

We will call the algebra $A_{c o}$ the cone algebra and the algebra $\mathrm{Cyl}(\mathbb{D})$ the cylinder algebra.

Copyright 2016 by the Tusi Mathematical Research Group.

Received Dec. 4, 2014; Accepted May 13, 2015.

${ }^{*}$ Corresponding author.

2010 Mathematics Subject Classification. Primary 46J10; Secondary 46J15, 46J20, 30H50.

Keywords. cone algebra, cylinder algebra, Bézout equation, maximal ideals, stable ranks. 


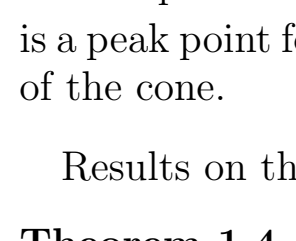

Ann. Funct. Anal. 7 (2016), no. 1, 42-60

http://dx.doi.org/10.1215/20088752-3163513

ISSN: 2008-8752 (electronic)

http://projecteuclid.org/afa

\title{
THE CONE AND CYLINDER ALGEBRAS
}

\author{
RAYMOND MORTINI $^{1^{*}}$ and RUDOLF RUPP ${ }^{2}$ \\ Dedicated to Professor Anthony To-Ming Lau
}

Communicated by J. Esterle

\begin{abstract}
In this exposition-type note we present detailed proofs of certain assertions concerning several algebraic properties of the cone and cylinder algebras. These include a determination of the maximal ideals, the solution of the Bézout equation, and a computation of the stable ranks by elementary methods.
\end{abstract}

\section{INTRODUCTION}

Let $\mathbb{D}=\{z \in \mathbb{C}:|z|<1\}$ be the open unit disk in the complex plane $\mathbb{C}$, and let $\mathbf{D}$ be its closure. As usual, $C(\mathbf{D}, \mathbb{C})$ denotes the space of continuous, complexvalued functions on $\mathbf{D}$ and $A(\mathbf{D})$ denotes the disk algebra, that is, the algebra of all functions in $C(\mathbf{D}, \mathbb{C})$ which are holomorphic in $\mathbb{D}$. By the Stone-Weierstrass theorem, we have $C(\mathbf{D}, \mathbb{C})=[z, \bar{z}]_{\text {alg }}$ and $A(\mathbf{D})=[z]_{\text {alg }}$, the uniformly closed subalgebras generated by $z, \bar{z}$ (resp., $z$ ) on $\mathbf{D}$. In this expositional note, we study the uniformly closed subalgebra

$$
A_{c o}=[z,|z|]_{\mathrm{alg}} \subseteq C(\mathbf{D}, \mathbb{C})
$$

of $C(\mathbf{D}, \mathbb{C})$, which is generated by $z$ and $|z|$, as well as by the algebra

$$
\operatorname{Cyl}(\mathbb{D})=\{f \in C(\mathbf{D} \times[0,1], \mathbb{C}): f(\cdot, t) \in A(\mathbf{D}) \text { for all } t \in[0,1]\} .
$$

We will call the algebra $A_{c o}$ the cone algebra and the algebra $\mathrm{Cyl}(\mathbb{D})$ the cylinder algebra.

Copyright 2016 by the Tusi Mathematical Research Group.

Received Dec. 4, 2014; Accepted May 13, 2015.

${ }^{*}$ Corresponding author.

2010 Mathematics Subject Classification. Primary 46J10; Secondary 46J15, 46J20, 30H50.

Keywords. cone algebra, cylinder algebra, Bézout equation, maximal ideals, stable ranks. 


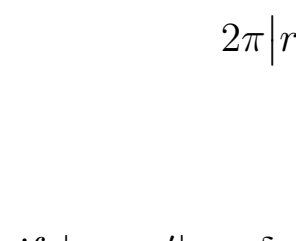

Ann. Funct. Anal. 7 (2016), no. 1, 42-60

http://dx.doi.org/10.1215/20088752-3163513

ISSN: 2008-8752 (electronic)

http://projecteuclid.org/afa

\title{
THE CONE AND CYLINDER ALGEBRAS
}

\author{
RAYMOND MORTINI $^{1^{*}}$ and RUDOLF RUPP ${ }^{2}$ \\ Dedicated to Professor Anthony To-Ming Lau
}

Communicated by J. Esterle

\begin{abstract}
In this exposition-type note we present detailed proofs of certain assertions concerning several algebraic properties of the cone and cylinder algebras. These include a determination of the maximal ideals, the solution of the Bézout equation, and a computation of the stable ranks by elementary methods.
\end{abstract}

\section{INTRODUCTION}

Let $\mathbb{D}=\{z \in \mathbb{C}:|z|<1\}$ be the open unit disk in the complex plane $\mathbb{C}$, and let $\mathbf{D}$ be its closure. As usual, $C(\mathbf{D}, \mathbb{C})$ denotes the space of continuous, complexvalued functions on $\mathbf{D}$ and $A(\mathbf{D})$ denotes the disk algebra, that is, the algebra of all functions in $C(\mathbf{D}, \mathbb{C})$ which are holomorphic in $\mathbb{D}$. By the Stone-Weierstrass theorem, we have $C(\mathbf{D}, \mathbb{C})=[z, \bar{z}]_{\text {alg }}$ and $A(\mathbf{D})=[z]_{\text {alg }}$, the uniformly closed subalgebras generated by $z, \bar{z}$ (resp., $z$ ) on $\mathbf{D}$. In this expositional note, we study the uniformly closed subalgebra

$$
A_{c o}=[z,|z|]_{\mathrm{alg}} \subseteq C(\mathbf{D}, \mathbb{C})
$$

of $C(\mathbf{D}, \mathbb{C})$, which is generated by $z$ and $|z|$, as well as by the algebra

$$
\operatorname{Cyl}(\mathbb{D})=\{f \in C(\mathbf{D} \times[0,1], \mathbb{C}): f(\cdot, t) \in A(\mathbf{D}) \text { for all } t \in[0,1]\} .
$$

We will call the algebra $A_{c o}$ the cone algebra and the algebra $\mathrm{Cyl}(\mathbb{D})$ the cylinder algebra.

Copyright 2016 by the Tusi Mathematical Research Group.

Received Dec. 4, 2014; Accepted May 13, 2015.

${ }^{*}$ Corresponding author.

2010 Mathematics Subject Classification. Primary 46J10; Secondary 46J15, 46J20, 30H50.

Keywords. cone algebra, cylinder algebra, Bézout equation, maximal ideals, stable ranks. 


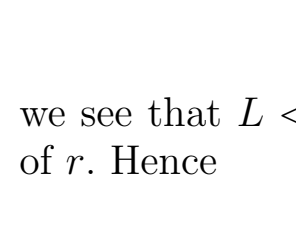

Ann. Funct. Anal. 7 (2016), no. 1, 42-60

http://dx.doi.org/10.1215/20088752-3163513

ISSN: 2008-8752 (electronic)

http://projecteuclid.org/afa

\title{
THE CONE AND CYLINDER ALGEBRAS
}

\author{
RAYMOND MORTINI $^{1^{*}}$ and RUDOLF RUPP ${ }^{2}$ \\ Dedicated to Professor Anthony To-Ming Lau
}

Communicated by J. Esterle

\begin{abstract}
In this exposition-type note we present detailed proofs of certain assertions concerning several algebraic properties of the cone and cylinder algebras. These include a determination of the maximal ideals, the solution of the Bézout equation, and a computation of the stable ranks by elementary methods.
\end{abstract}

\section{INTRODUCTION}

Let $\mathbb{D}=\{z \in \mathbb{C}:|z|<1\}$ be the open unit disk in the complex plane $\mathbb{C}$, and let $\mathbf{D}$ be its closure. As usual, $C(\mathbf{D}, \mathbb{C})$ denotes the space of continuous, complexvalued functions on $\mathbf{D}$ and $A(\mathbf{D})$ denotes the disk algebra, that is, the algebra of all functions in $C(\mathbf{D}, \mathbb{C})$ which are holomorphic in $\mathbb{D}$. By the Stone-Weierstrass theorem, we have $C(\mathbf{D}, \mathbb{C})=[z, \bar{z}]_{\text {alg }}$ and $A(\mathbf{D})=[z]_{\text {alg }}$, the uniformly closed subalgebras generated by $z, \bar{z}$ (resp., $z$ ) on $\mathbf{D}$. In this expositional note, we study the uniformly closed subalgebra

$$
A_{c o}=[z,|z|]_{\mathrm{alg}} \subseteq C(\mathbf{D}, \mathbb{C})
$$

of $C(\mathbf{D}, \mathbb{C})$, which is generated by $z$ and $|z|$, as well as by the algebra

$$
\operatorname{Cyl}(\mathbb{D})=\{f \in C(\mathbf{D} \times[0,1], \mathbb{C}): f(\cdot, t) \in A(\mathbf{D}) \text { for all } t \in[0,1]\} .
$$

We will call the algebra $A_{c o}$ the cone algebra and the algebra $\mathrm{Cyl}(\mathbb{D})$ the cylinder algebra.

Copyright 2016 by the Tusi Mathematical Research Group.

Received Dec. 4, 2014; Accepted May 13, 2015.

${ }^{*}$ Corresponding author.

2010 Mathematics Subject Classification. Primary 46J10; Secondary 46J15, 46J20, 30H50.

Keywords. cone algebra, cylinder algebra, Bézout equation, maximal ideals, stable ranks. 


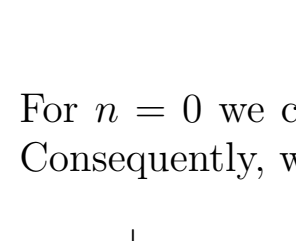

Ann. Funct. Anal. 7 (2016), no. 1, 42-60

http://dx.doi.org/10.1215/20088752-3163513

ISSN: 2008-8752 (electronic)

http://projecteuclid.org/afa

\title{
THE CONE AND CYLINDER ALGEBRAS
}

\author{
RAYMOND MORTINI $^{1^{*}}$ and RUDOLF RUPP ${ }^{2}$ \\ Dedicated to Professor Anthony To-Ming Lau
}

Communicated by J. Esterle

\begin{abstract}
In this exposition-type note we present detailed proofs of certain assertions concerning several algebraic properties of the cone and cylinder algebras. These include a determination of the maximal ideals, the solution of the Bézout equation, and a computation of the stable ranks by elementary methods.
\end{abstract}

\section{INTRODUCTION}

Let $\mathbb{D}=\{z \in \mathbb{C}:|z|<1\}$ be the open unit disk in the complex plane $\mathbb{C}$, and let $\mathbf{D}$ be its closure. As usual, $C(\mathbf{D}, \mathbb{C})$ denotes the space of continuous, complexvalued functions on $\mathbf{D}$ and $A(\mathbf{D})$ denotes the disk algebra, that is, the algebra of all functions in $C(\mathbf{D}, \mathbb{C})$ which are holomorphic in $\mathbb{D}$. By the Stone-Weierstrass theorem, we have $C(\mathbf{D}, \mathbb{C})=[z, \bar{z}]_{\text {alg }}$ and $A(\mathbf{D})=[z]_{\text {alg }}$, the uniformly closed subalgebras generated by $z, \bar{z}$ (resp., $z$ ) on $\mathbf{D}$. In this expositional note, we study the uniformly closed subalgebra

$$
A_{c o}=[z,|z|]_{\mathrm{alg}} \subseteq C(\mathbf{D}, \mathbb{C})
$$

of $C(\mathbf{D}, \mathbb{C})$, which is generated by $z$ and $|z|$, as well as by the algebra

$$
\operatorname{Cyl}(\mathbb{D})=\{f \in C(\mathbf{D} \times[0,1], \mathbb{C}): f(\cdot, t) \in A(\mathbf{D}) \text { for all } t \in[0,1]\} .
$$

We will call the algebra $A_{c o}$ the cone algebra and the algebra $\mathrm{Cyl}(\mathbb{D})$ the cylinder algebra.

Copyright 2016 by the Tusi Mathematical Research Group.

Received Dec. 4, 2014; Accepted May 13, 2015.

${ }^{*}$ Corresponding author.

2010 Mathematics Subject Classification. Primary 46J10; Secondary 46J15, 46J20, 30H50.

Keywords. cone algebra, cylinder algebra, Bézout equation, maximal ideals, stable ranks. 


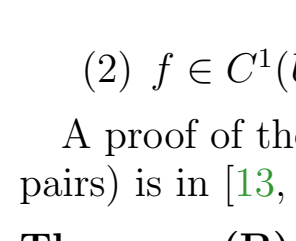

Ann. Funct. Anal. 7 (2016), no. 1, 42-60

http://dx.doi.org/10.1215/20088752-3163513

ISSN: 2008-8752 (electronic)

http://projecteuclid.org/afa

\title{
THE CONE AND CYLINDER ALGEBRAS
}

\author{
RAYMOND MORTINI $^{1^{*}}$ and RUDOLF RUPP ${ }^{2}$ \\ Dedicated to Professor Anthony To-Ming Lau
}

Communicated by J. Esterle

\begin{abstract}
In this exposition-type note we present detailed proofs of certain assertions concerning several algebraic properties of the cone and cylinder algebras. These include a determination of the maximal ideals, the solution of the Bézout equation, and a computation of the stable ranks by elementary methods.
\end{abstract}

\section{INTRODUCTION}

Let $\mathbb{D}=\{z \in \mathbb{C}:|z|<1\}$ be the open unit disk in the complex plane $\mathbb{C}$, and let $\mathbf{D}$ be its closure. As usual, $C(\mathbf{D}, \mathbb{C})$ denotes the space of continuous, complexvalued functions on $\mathbf{D}$ and $A(\mathbf{D})$ denotes the disk algebra, that is, the algebra of all functions in $C(\mathbf{D}, \mathbb{C})$ which are holomorphic in $\mathbb{D}$. By the Stone-Weierstrass theorem, we have $C(\mathbf{D}, \mathbb{C})=[z, \bar{z}]_{\text {alg }}$ and $A(\mathbf{D})=[z]_{\text {alg }}$, the uniformly closed subalgebras generated by $z, \bar{z}$ (resp., $z$ ) on $\mathbf{D}$. In this expositional note, we study the uniformly closed subalgebra

$$
A_{c o}=[z,|z|]_{\mathrm{alg}} \subseteq C(\mathbf{D}, \mathbb{C})
$$

of $C(\mathbf{D}, \mathbb{C})$, which is generated by $z$ and $|z|$, as well as by the algebra

$$
\operatorname{Cyl}(\mathbb{D})=\{f \in C(\mathbf{D} \times[0,1], \mathbb{C}): f(\cdot, t) \in A(\mathbf{D}) \text { for all } t \in[0,1]\} .
$$

We will call the algebra $A_{c o}$ the cone algebra and the algebra $\mathrm{Cyl}(\mathbb{D})$ the cylinder algebra.

Copyright 2016 by the Tusi Mathematical Research Group.

Received Dec. 4, 2014; Accepted May 13, 2015.

${ }^{*}$ Corresponding author.

2010 Mathematics Subject Classification. Primary 46J10; Secondary 46J15, 46J20, 30H50.

Keywords. cone algebra, cylinder algebra, Bézout equation, maximal ideals, stable ranks. 


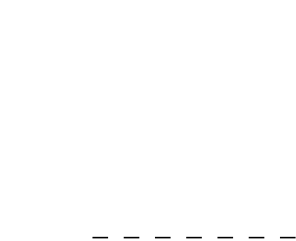

Ann. Funct. Anal. 7 (2016), no. 1, 42-60

http://dx.doi.org/10.1215/20088752-3163513

ISSN: 2008-8752 (electronic)

http://projecteuclid.org/afa

\title{
THE CONE AND CYLINDER ALGEBRAS
}

\author{
RAYMOND MORTINI $^{1^{*}}$ and RUDOLF RUPP ${ }^{2}$ \\ Dedicated to Professor Anthony To-Ming Lau
}

Communicated by J. Esterle

\begin{abstract}
In this exposition-type note we present detailed proofs of certain assertions concerning several algebraic properties of the cone and cylinder algebras. These include a determination of the maximal ideals, the solution of the Bézout equation, and a computation of the stable ranks by elementary methods.
\end{abstract}

\section{INTRODUCTION}

Let $\mathbb{D}=\{z \in \mathbb{C}:|z|<1\}$ be the open unit disk in the complex plane $\mathbb{C}$, and let $\mathbf{D}$ be its closure. As usual, $C(\mathbf{D}, \mathbb{C})$ denotes the space of continuous, complexvalued functions on $\mathbf{D}$ and $A(\mathbf{D})$ denotes the disk algebra, that is, the algebra of all functions in $C(\mathbf{D}, \mathbb{C})$ which are holomorphic in $\mathbb{D}$. By the Stone-Weierstrass theorem, we have $C(\mathbf{D}, \mathbb{C})=[z, \bar{z}]_{\text {alg }}$ and $A(\mathbf{D})=[z]_{\text {alg }}$, the uniformly closed subalgebras generated by $z, \bar{z}$ (resp., $z$ ) on $\mathbf{D}$. In this expositional note, we study the uniformly closed subalgebra

$$
A_{c o}=[z,|z|]_{\mathrm{alg}} \subseteq C(\mathbf{D}, \mathbb{C})
$$

of $C(\mathbf{D}, \mathbb{C})$, which is generated by $z$ and $|z|$, as well as by the algebra

$$
\operatorname{Cyl}(\mathbb{D})=\{f \in C(\mathbf{D} \times[0,1], \mathbb{C}): f(\cdot, t) \in A(\mathbf{D}) \text { for all } t \in[0,1]\} .
$$

We will call the algebra $A_{c o}$ the cone algebra and the algebra $\mathrm{Cyl}(\mathbb{D})$ the cylinder algebra.

Copyright 2016 by the Tusi Mathematical Research Group.

Received Dec. 4, 2014; Accepted May 13, 2015.

${ }^{*}$ Corresponding author.

2010 Mathematics Subject Classification. Primary 46J10; Secondary 46J15, 46J20, 30H50.

Keywords. cone algebra, cylinder algebra, Bézout equation, maximal ideals, stable ranks. 


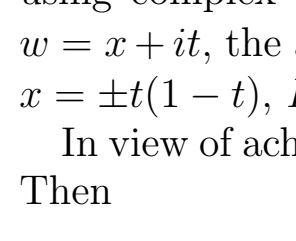

Ann. Funct. Anal. 7 (2016), no. 1, 42-60

http://dx.doi.org/10.1215/20088752-3163513

ISSN: 2008-8752 (electronic)

http://projecteuclid.org/afa

\title{
THE CONE AND CYLINDER ALGEBRAS
}

\author{
RAYMOND MORTINI $^{1^{*}}$ and RUDOLF RUPP ${ }^{2}$ \\ Dedicated to Professor Anthony To-Ming Lau
}

Communicated by J. Esterle

\begin{abstract}
In this exposition-type note we present detailed proofs of certain assertions concerning several algebraic properties of the cone and cylinder algebras. These include a determination of the maximal ideals, the solution of the Bézout equation, and a computation of the stable ranks by elementary methods.
\end{abstract}

\section{INTRODUCTION}

Let $\mathbb{D}=\{z \in \mathbb{C}:|z|<1\}$ be the open unit disk in the complex plane $\mathbb{C}$, and let $\mathbf{D}$ be its closure. As usual, $C(\mathbf{D}, \mathbb{C})$ denotes the space of continuous, complexvalued functions on $\mathbf{D}$ and $A(\mathbf{D})$ denotes the disk algebra, that is, the algebra of all functions in $C(\mathbf{D}, \mathbb{C})$ which are holomorphic in $\mathbb{D}$. By the Stone-Weierstrass theorem, we have $C(\mathbf{D}, \mathbb{C})=[z, \bar{z}]_{\text {alg }}$ and $A(\mathbf{D})=[z]_{\text {alg }}$, the uniformly closed subalgebras generated by $z, \bar{z}$ (resp., $z$ ) on $\mathbf{D}$. In this expositional note, we study the uniformly closed subalgebra

$$
A_{c o}=[z,|z|]_{\mathrm{alg}} \subseteq C(\mathbf{D}, \mathbb{C})
$$

of $C(\mathbf{D}, \mathbb{C})$, which is generated by $z$ and $|z|$, as well as by the algebra

$$
\operatorname{Cyl}(\mathbb{D})=\{f \in C(\mathbf{D} \times[0,1], \mathbb{C}): f(\cdot, t) \in A(\mathbf{D}) \text { for all } t \in[0,1]\} .
$$

We will call the algebra $A_{c o}$ the cone algebra and the algebra $\mathrm{Cyl}(\mathbb{D})$ the cylinder algebra.

Copyright 2016 by the Tusi Mathematical Research Group.

Received Dec. 4, 2014; Accepted May 13, 2015.

${ }^{*}$ Corresponding author.

2010 Mathematics Subject Classification. Primary 46J10; Secondary 46J15, 46J20, 30H50.

Keywords. cone algebra, cylinder algebra, Bézout equation, maximal ideals, stable ranks. 


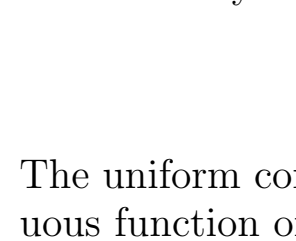

Ann. Funct. Anal. 7 (2016), no. 1, 42-60

http://dx.doi.org/10.1215/20088752-3163513

ISSN: 2008-8752 (electronic)

http://projecteuclid.org/afa

\title{
THE CONE AND CYLINDER ALGEBRAS
}

\author{
RAYMOND MORTINI $^{1^{*}}$ and RUDOLF RUPP ${ }^{2}$ \\ Dedicated to Professor Anthony To-Ming Lau
}

Communicated by J. Esterle

\begin{abstract}
In this exposition-type note we present detailed proofs of certain assertions concerning several algebraic properties of the cone and cylinder algebras. These include a determination of the maximal ideals, the solution of the Bézout equation, and a computation of the stable ranks by elementary methods.
\end{abstract}

\section{INTRODUCTION}

Let $\mathbb{D}=\{z \in \mathbb{C}:|z|<1\}$ be the open unit disk in the complex plane $\mathbb{C}$, and let $\mathbf{D}$ be its closure. As usual, $C(\mathbf{D}, \mathbb{C})$ denotes the space of continuous, complexvalued functions on $\mathbf{D}$ and $A(\mathbf{D})$ denotes the disk algebra, that is, the algebra of all functions in $C(\mathbf{D}, \mathbb{C})$ which are holomorphic in $\mathbb{D}$. By the Stone-Weierstrass theorem, we have $C(\mathbf{D}, \mathbb{C})=[z, \bar{z}]_{\text {alg }}$ and $A(\mathbf{D})=[z]_{\text {alg }}$, the uniformly closed subalgebras generated by $z, \bar{z}$ (resp., $z$ ) on $\mathbf{D}$. In this expositional note, we study the uniformly closed subalgebra

$$
A_{c o}=[z,|z|]_{\mathrm{alg}} \subseteq C(\mathbf{D}, \mathbb{C})
$$

of $C(\mathbf{D}, \mathbb{C})$, which is generated by $z$ and $|z|$, as well as by the algebra

$$
\operatorname{Cyl}(\mathbb{D})=\{f \in C(\mathbf{D} \times[0,1], \mathbb{C}): f(\cdot, t) \in A(\mathbf{D}) \text { for all } t \in[0,1]\} .
$$

We will call the algebra $A_{c o}$ the cone algebra and the algebra $\mathrm{Cyl}(\mathbb{D})$ the cylinder algebra.

Copyright 2016 by the Tusi Mathematical Research Group.

Received Dec. 4, 2014; Accepted May 13, 2015.

${ }^{*}$ Corresponding author.

2010 Mathematics Subject Classification. Primary 46J10; Secondary 46J15, 46J20, 30H50.

Keywords. cone algebra, cylinder algebra, Bézout equation, maximal ideals, stable ranks. 


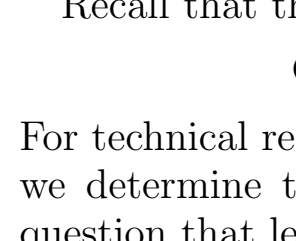

Ann. Funct. Anal. 7 (2016), no. 1, 42-60

http://dx.doi.org/10.1215/20088752-3163513

ISSN: 2008-8752 (electronic)

http://projecteuclid.org/afa

\title{
THE CONE AND CYLINDER ALGEBRAS
}

\author{
RAYMOND MORTINI $^{1^{*}}$ and RUDOLF RUPP ${ }^{2}$ \\ Dedicated to Professor Anthony To-Ming Lau
}

Communicated by J. Esterle

\begin{abstract}
In this exposition-type note we present detailed proofs of certain assertions concerning several algebraic properties of the cone and cylinder algebras. These include a determination of the maximal ideals, the solution of the Bézout equation, and a computation of the stable ranks by elementary methods.
\end{abstract}

\section{INTRODUCTION}

Let $\mathbb{D}=\{z \in \mathbb{C}:|z|<1\}$ be the open unit disk in the complex plane $\mathbb{C}$, and let $\mathbf{D}$ be its closure. As usual, $C(\mathbf{D}, \mathbb{C})$ denotes the space of continuous, complexvalued functions on $\mathbf{D}$ and $A(\mathbf{D})$ denotes the disk algebra, that is, the algebra of all functions in $C(\mathbf{D}, \mathbb{C})$ which are holomorphic in $\mathbb{D}$. By the Stone-Weierstrass theorem, we have $C(\mathbf{D}, \mathbb{C})=[z, \bar{z}]_{\text {alg }}$ and $A(\mathbf{D})=[z]_{\text {alg }}$, the uniformly closed subalgebras generated by $z, \bar{z}$ (resp., $z$ ) on $\mathbf{D}$. In this expositional note, we study the uniformly closed subalgebra

$$
A_{c o}=[z,|z|]_{\mathrm{alg}} \subseteq C(\mathbf{D}, \mathbb{C})
$$

of $C(\mathbf{D}, \mathbb{C})$, which is generated by $z$ and $|z|$, as well as by the algebra

$$
\operatorname{Cyl}(\mathbb{D})=\{f \in C(\mathbf{D} \times[0,1], \mathbb{C}): f(\cdot, t) \in A(\mathbf{D}) \text { for all } t \in[0,1]\} .
$$

We will call the algebra $A_{c o}$ the cone algebra and the algebra $\mathrm{Cyl}(\mathbb{D})$ the cylinder algebra.

Copyright 2016 by the Tusi Mathematical Research Group.

Received Dec. 4, 2014; Accepted May 13, 2015.

${ }^{*}$ Corresponding author.

2010 Mathematics Subject Classification. Primary 46J10; Secondary 46J15, 46J20, 30H50.

Keywords. cone algebra, cylinder algebra, Bézout equation, maximal ideals, stable ranks. 


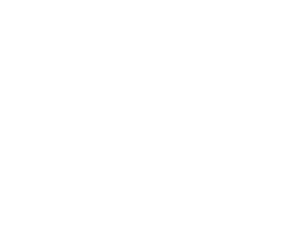

Ann. Funct. Anal. 7 (2016), no. 1, 42-60

http://dx.doi.org/10.1215/20088752-3163513

ISSN: 2008-8752 (electronic)

http://projecteuclid.org/afa

\title{
THE CONE AND CYLINDER ALGEBRAS
}

\author{
RAYMOND MORTINI $^{1^{*}}$ and RUDOLF RUPP ${ }^{2}$ \\ Dedicated to Professor Anthony To-Ming Lau
}

Communicated by J. Esterle

\begin{abstract}
In this exposition-type note we present detailed proofs of certain assertions concerning several algebraic properties of the cone and cylinder algebras. These include a determination of the maximal ideals, the solution of the Bézout equation, and a computation of the stable ranks by elementary methods.
\end{abstract}

\section{INTRODUCTION}

Let $\mathbb{D}=\{z \in \mathbb{C}:|z|<1\}$ be the open unit disk in the complex plane $\mathbb{C}$, and let $\mathbf{D}$ be its closure. As usual, $C(\mathbf{D}, \mathbb{C})$ denotes the space of continuous, complexvalued functions on $\mathbf{D}$ and $A(\mathbf{D})$ denotes the disk algebra, that is, the algebra of all functions in $C(\mathbf{D}, \mathbb{C})$ which are holomorphic in $\mathbb{D}$. By the Stone-Weierstrass theorem, we have $C(\mathbf{D}, \mathbb{C})=[z, \bar{z}]_{\text {alg }}$ and $A(\mathbf{D})=[z]_{\text {alg }}$, the uniformly closed subalgebras generated by $z, \bar{z}$ (resp., $z$ ) on $\mathbf{D}$. In this expositional note, we study the uniformly closed subalgebra

$$
A_{c o}=[z,|z|]_{\mathrm{alg}} \subseteq C(\mathbf{D}, \mathbb{C})
$$

of $C(\mathbf{D}, \mathbb{C})$, which is generated by $z$ and $|z|$, as well as by the algebra

$$
\operatorname{Cyl}(\mathbb{D})=\{f \in C(\mathbf{D} \times[0,1], \mathbb{C}): f(\cdot, t) \in A(\mathbf{D}) \text { for all } t \in[0,1]\} .
$$

We will call the algebra $A_{c o}$ the cone algebra and the algebra $\mathrm{Cyl}(\mathbb{D})$ the cylinder algebra.

Copyright 2016 by the Tusi Mathematical Research Group.

Received Dec. 4, 2014; Accepted May 13, 2015.

${ }^{*}$ Corresponding author.

2010 Mathematics Subject Classification. Primary 46J10; Secondary 46J15, 46J20, 30H50.

Keywords. cone algebra, cylinder algebra, Bézout equation, maximal ideals, stable ranks. 


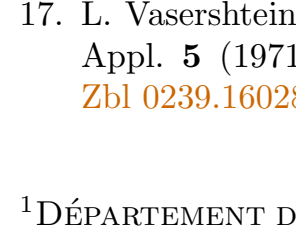

Ann. Funct. Anal. 7 (2016), no. 1, 42-60

http://dx.doi.org/10.1215/20088752-3163513

ISSN: 2008-8752 (electronic)

http://projecteuclid.org/afa

\title{
THE CONE AND CYLINDER ALGEBRAS
}

\author{
RAYMOND MORTINI $^{1^{*}}$ and RUDOLF RUPP ${ }^{2}$ \\ Dedicated to Professor Anthony To-Ming Lau
}

Communicated by J. Esterle

\begin{abstract}
In this exposition-type note we present detailed proofs of certain assertions concerning several algebraic properties of the cone and cylinder algebras. These include a determination of the maximal ideals, the solution of the Bézout equation, and a computation of the stable ranks by elementary methods.
\end{abstract}

\section{INTRODUCTION}

Let $\mathbb{D}=\{z \in \mathbb{C}:|z|<1\}$ be the open unit disk in the complex plane $\mathbb{C}$, and let $\mathbf{D}$ be its closure. As usual, $C(\mathbf{D}, \mathbb{C})$ denotes the space of continuous, complexvalued functions on $\mathbf{D}$ and $A(\mathbf{D})$ denotes the disk algebra, that is, the algebra of all functions in $C(\mathbf{D}, \mathbb{C})$ which are holomorphic in $\mathbb{D}$. By the Stone-Weierstrass theorem, we have $C(\mathbf{D}, \mathbb{C})=[z, \bar{z}]_{\text {alg }}$ and $A(\mathbf{D})=[z]_{\text {alg }}$, the uniformly closed subalgebras generated by $z, \bar{z}$ (resp., $z$ ) on $\mathbf{D}$. In this expositional note, we study the uniformly closed subalgebra

$$
A_{c o}=[z,|z|]_{\mathrm{alg}} \subseteq C(\mathbf{D}, \mathbb{C})
$$

of $C(\mathbf{D}, \mathbb{C})$, which is generated by $z$ and $|z|$, as well as by the algebra

$$
\operatorname{Cyl}(\mathbb{D})=\{f \in C(\mathbf{D} \times[0,1], \mathbb{C}): f(\cdot, t) \in A(\mathbf{D}) \text { for all } t \in[0,1]\} .
$$

We will call the algebra $A_{c o}$ the cone algebra and the algebra $\mathrm{Cyl}(\mathbb{D})$ the cylinder algebra.

Copyright 2016 by the Tusi Mathematical Research Group.

Received Dec. 4, 2014; Accepted May 13, 2015.

${ }^{*}$ Corresponding author.

2010 Mathematics Subject Classification. Primary 46J10; Secondary 46J15, 46J20, 30H50.

Keywords. cone algebra, cylinder algebra, Bézout equation, maximal ideals, stable ranks. 